\title{
GSTM1 null polymorphism at the glutathione S-transferase $M 1$ locus: phenotype and genotype studies in patients with primary biliary cirrhosis
}

\author{
M H Davies, E Elias, S Acharya, W Cotton, G C Faulder, A A Fryer, R C Strange
}

\begin{abstract}
Studies were carried out to test the hypothesis that the GSTM1 null phenotype at the $\mu(\mathbf{m u})$ class glutathione $S$-transferase 1 locus is associated with an increased predisposition to primary biliary cirrhosis. Starch gel electrophoresis was used to compare the prevalence of GSTM1 null phenotype 0 in patients with end stage primary biliary cirrhosis and a group of controls without evidence of liver disease. The prevalence of GSTM1 null phenotype in the primary biliary cirrhosis and control groups was similar; $39 \%$ and $45 \%$ respectively. In the primary biliary cirrhosis group all subjects were of the common GSTM1 0, GSTM1 A, GSTM1 B or GSTM1 A, B phenotypes while in the controls, one subject showed an isoform with an anodal mobility compatible with it being a product of the putative GSTM1*3 allele. As the GSTM1 phenotype might be changed by the disease process, the polymerase chain reaction was used to amplify the exon 4-exon 5 region of GSTM1 and show that in 13 control subjects and 11 patients with primary biliary cirrhosis, GSTM1 positive and negative genotypes were associated with corresponding GSTM1 expressing and nonexpressing phenotypes respectively. The control subject with GSTM1 3 phenotype showed a positive genotype.

(Gut 1993; 34: 549-553)
\end{abstract}

The hepatic response to many chemicals is polymorphic. ${ }^{1-3}$ The phenomenon partly results from multiple allelism at loci encoding phase I and II drug metabolising enzymes and includes examples of inter-individual variation in metabolism such as the occurrence of hypotension in poor metabolisers of debrisoquine. ${ }^{2}$ Liver diseases associated with autoantibodies may also be precipitated by chemicals such as tienilic acid (Ticrynafen) which can induce a specific form of chronic active hepatitis. Idiosyncratic metabolism of the drug is believed to trigger the disease process. ${ }^{+5}$

Primary biliary cirrhosis is a disease of unknown cause and widely varying severity that seems to result from both genetical and environmental factors. Dominant features are the presence of circulating antimitochondrial antibodies and damage and destruction of intrahepatic ducts, a process associated with infiltrating, chronic inflammatory cells, and ductular proliferation followed by scarring. The mechanism of breakdown in immune tolerance is unknown but less effective detoxication of a xenobiotic that damages ductal antigens may be a factor in determining susceptibility. Ineffective detoxication of the reactive oxygen species produced during inflammation may also be important in determining disease aggressiveness.

Support for the view that deficient detoxication of a xenobiotic(s) is associated with a predisposition to primary biliary cirrhosis comes from the recognised predominance of female patients with the disease which is compatible with oestrogen mediated effects on the expression of detoxicating enzymes. Further, poor sulphoxidation has been described in patients with primary biliary cirrhosis. ${ }^{6}$ This deficiency probably results from reduced activity of cysteine dioxygenase, a polymorphic enzyme that catalyses the conversion of cysteine to sulphate.

While it is not known if polymorphisms of other detoxicating enzymes are associated with primary biliary cirrhosis, enzymes such as the glutathione S-transferase (GST) that catalyse the conjugation of many electrophiles with reduced glutathione as well as the repair of DNA and lipid oxidised by reactive oxygen species are possible candidates. The main GST isoforms are classified into $\alpha$ (alpha), $\mu$ (mu), $\theta$ (theta), and $\pi$ (pi) classes. $^{7-9}$ The most widely expressed $\mu$ gene, GSTM1, is polymorphic with four common phenotypes resulting from homo and heterozygotic combinations of the GSTM1 ${ }^{\star} 0$ $\left(\mathrm{GST} 1^{\star} 0\right), \quad \mathrm{GSTM} 1^{\star} \mathrm{A} \quad\left(\mathrm{GST} 1^{\star} 2\right)$, and GSTM1 ${ }^{\star} B\left(G S T 1{ }^{\star} 1\right)$ alleles. ${ }^{910}$ About $50 \%$ of most populations are homozygous for the deleted GSTM ${ }^{\star} 0$ allele and express no GSTM1 enzyme. ${ }^{9-11}$ A further, rare putative allele, GSTM1 ${ }^{\star} 3$, has been reported but unlike GSTM ${ }^{\star}{ }^{\star} A$ and GSTM $1{ }^{\star} B$ has not been characterised. ${ }^{12} 13$

The importance of GSTM1 in the detoxication of electrophiles is indicated by the increased occurrence of GSTM1 null phenotype (GSTM1 0 ) in several malignancies ${ }^{1121+16}$ and data showing GSTM1 0 lymphocytes are more sensitive to epoxide induced sister chromatid exchange than GSTM1 expressing cells. ${ }^{17}$ The significance of the GSTM1 0 genotype in determining susceptibility to carcinogens is unclear, however, because only a small number of malignancies have been studied and often with a small number of patients. ${ }^{12} 1618$ Furthermore, data in lung cancer are conflicting ${ }^{11}$ and no relation seems to exist between GSTM1 0 and at least some malignancies. ${ }^{18} 19$

GSTM1 may also be important in the repair of DNA damaged by reactive oxygen species because in vitro substrates include 5-hydroperoxymethyluracil and DNA hydroperoxide. ${ }^{20}$ Studies showing that GSTM1 is generally 
expressed in cells with a high metabolic activity and that hepatic expression is up regulated during human development in preparation for the relatively hyperoxic, extrauterine environment, also suggest these enzymes are part of an antioxidant defence system. ${ }^{21}$

The comparatively small amount of data on GSTM1 expression in patients with liver disease are confused. Thus, an increased occurrence of GSTM 10 has been reported in a small series of Japanese patients with inflammatory conditions, chronic hepatitis, and alcoholic liver disease ${ }^{12}$ and, also in Russian patients suffering alcoholic hepatitis. ${ }^{22}$ The occurrence of GSTM1 0 however, was not found increased in white French subjects with alcoholic cirrhosis. ${ }^{23}$

Impaired ability to detoxicate xenobiotics and reactive oxygen species, or both, may increase susceptibility to primary biliary cirrhosis. GSTM1 0 might result in impaired detoxication of antigen inducing xenobiotics so increasing susceptibility to the disease or, in reduced ability to repair DNA oxidised during inflammation, resulting in more aggressive disease. We describe studies using starch gel electrophoresis and the polymerase chain reaction to determine the prevalence of GSTM1 0 in controls and subjects with the disease.

\section{Patients and methods}

\section{LIVER SAMPLES}

GSTM1 phenotype was determined in liver samples from 44 female and four male patients with primary biliary cirrhosis. The diagnosis of primary biliary cirrhosis was made on the basis of positive anti-mitochondrial antibodies, consistent histological change, and cholestatic biochemical dysfunction. All patients had end stage liver disease as a result of aggressive primary biliary cirrhosis and required an orthotopic liver transplant. Liver samples were obtained immediately after hepatectomy, liver parenchyma was dissected, snap frozen in liquid $\mathrm{N}_{2}$ and stored at $-70^{\circ} \mathrm{C}$ until analysis. Control liver tissue was obtained from surplus donor livers (12 men, eight women) following cut down for paediatric liver transplantation and 49 subjects at postmortem examination. The controls had no history of liver disease and were without evidence of malignancy.

\section{DETERMINATION OF GSTMI PHENOTYPE}

The GSTM1 phenotype was determined using horizontal starch gel electrophoresis. ${ }^{10}$ Cytosols, prepared by homogenising liver pieces in $20 \mathrm{mM}$ TRIS-HCl buffer ( $\mathrm{pH} 7 \cdot 2$ ), centrifuging the homogenate $\left(60 \mathrm{~min}, 4^{\circ} \mathrm{C}, 150000 \mathrm{~g}\right)$, and recovering the supernatant (cytosol), were electrophoresed $\left(16 \mathrm{~h}, 4^{\circ} \mathrm{C}\right)$ in starch gels (length

TABLE Frequency of GSTM1 phenotypes in controls and patients with primary biliary cirrhosis

\begin{tabular}{lllllll}
\hline Phenotype & $\begin{array}{l}\text { GSTM10 } \\
(\%)\end{array}$ & $\begin{array}{l}\text { GSTM1 B } \\
(\%)\end{array}$ & $\begin{array}{l}\text { GSTM1 A } \\
(\%)\end{array}$ & $\begin{array}{l}\text { GSTM1 A,B } \\
(\%)\end{array}$ & $\begin{array}{l}\text { GSTM1 3 } \\
(\%)\end{array}$ & Total \\
\hline $\begin{array}{l}\text { Controls } \\
\text { Primary } \\
\text { biliary } \\
\text { cirrhosis }\end{array}$ & $31(45)$ & $13(19)$ & $20(29)$ & $4(6)$ & $1(1)$ & 69 \\
\hline
\end{tabular}

$19 \mathrm{~cm}$, width $11 \mathrm{~cm}$, depth $1 \mathrm{~cm}$ ) using a TRIScitrate buffer system (pH 7.5) GST isoenzymes were visualised using an initial agarose overlay containing 1-chloro-2,4-dinitrobenzene and reduced glutathione followed by a second overlay containing an iodine-potassium iodide solution.

\section{DETERMINATION OF GSTMI GENOTYPE}

GSTM1 genotype was determined in DNA extracted from liver samples ${ }^{24}$ using polymerase chain reaction performed with primers to exon 4 (5'-CTGCCCTACTTGATTGATGGG-3') and exon 5 (5'-CTGGATTGTAGCAGATCATGC$\left.3^{\prime}\right)$ of GSTM $1^{19}$ and, as internal positive control, primers (5'-ACACAACTGTGTTCACTAGC-3' and 5'-CAACTTCATCCACGTTCACC-3') to the $\beta$ globin gene. ${ }^{25}$ Polymerase chain reactions were carried out in duplicate in a solution $(50 \mu \mathrm{l})$ containing the primers $(4 \times 500 \mathrm{nM})$, Taq polymerase (1 unit), dNTP $(4 \times 0.2 \mathrm{mM})$, target DNA $(0.5 \mu \mathrm{g})$, and buffer (10 mM TRIS-HCl, $\mathrm{pH} 9 \cdot 0,50 \mathrm{mM} \mathrm{KCl}, 0 \cdot 1 \% \mathrm{vol} / \mathrm{vol}$ Triton X-100, $1.5 \mathrm{mM} \mathrm{MgCl}_{2}$ ). The reaction mixture was overlayed with $50 \mu \mathrm{l}$ mineral oil to prevent evaporation. After initial denaturation at $94^{\circ} \mathrm{C}$ for three minutes, 30 cycles of primer annealing $\left(55^{\circ} \mathrm{C}, 1\right.$ $\mathrm{min})$, elongation $\left(72^{\circ} \mathrm{C}, 2 \mathrm{~min}\right)$, and denaturation $\left(94^{\circ} \mathrm{C}, 1 \mathrm{~min}\right.$ ) were performed (Omnigene Thermal Cycler, Hybaid Ltd, Teddington, Middlesex). Polymerase chain reaction products were resolved by electrophoresis in $2 \% \mathrm{vol} / \mathrm{vol}$ agarose gels, stained with ethidium bromide, and photographed under ultraviolet light.

As GSTM 1 is deleted in GSTM $1{ }^{\star} 0 / \mathrm{GSTM} 1{ }^{\star} 0$ homozygotes, " this genotype can be differentiated from GSTM ${ }^{\star} 0$ heterozygotes, GSTM ${ }^{\star}{ }^{\star A}$, and GSTM1^B homo and heterozygotes by the absence of a $273 \mathrm{bp}$ fragment (exon 4, intron 4, exon 5 gene segment) on agarose gels. GSTM $1^{\star} A$ and GSTM ${ }^{\star}{ }^{\star} B$ differ by a single base in exon 7 and so are detected equally. ${ }^{26}{ }^{27}$ Success of the polymerase chain reaction was confirmed by the amplification of a $110 \mathrm{bp}$ product from the $\beta$ globin gene.

\section{Results}

GSTM1 PHENOTYPES IN CONTROLS AND PATIENTS WITH PRIMARY BILIARY CIRRHOSIS

GST isoenzymes in liver samples were resolved by electrophoresis in starch gels into four sets; fast anodal mobility ( $\pi$ class), slower anodal mobility ( $\alpha$ class isoform termed GST2 (5.5)), ${ }^{16}$ slow anodal mobility ( $\mu$ class, various GSTM 1 isoenzymes), and cathodal mobility ( $\alpha$ class) (Fig 1). There were no apparent differences between controls and patients with primary biliary cirrhosis in the patterns or comparative values of expression of these GST classes.

While there is often considerable between individual variation in the number of $\alpha$ class isoenzymes expressed in liver cytosols (Fig 1), these enzymes are usually strongly expressed. Evidence of $\alpha$ class activity was used therefore, as a check on the quality of specimens. All samples assigned a GSTM1 0 phenotype showed cathodally migrating $\alpha$ class GST activity. 
Figure 1: Starch gel electrophoresis of liver cytosols from controls and patients with primary biliary cirrhosis. The gel shows the origin and the migration of various $\alpha$ class $G S T$ towards the cathode

(bottom). Various GST isoforms migrated towards the anode (top); $\mu$ class isoforms with intermediate anodal mobility, the $\alpha$ class isoform termed GST2 (5.5), and $\pi$ class isoforms with fast anodal mobility (top). The GSTM 1 phenotypes are (left to right): GSTM $1 B$

(control), GSTM 10

(PBC), GSTMI B (PBC), GSTM $1 A, B(P B C)$, and putative GSTM13

(control).

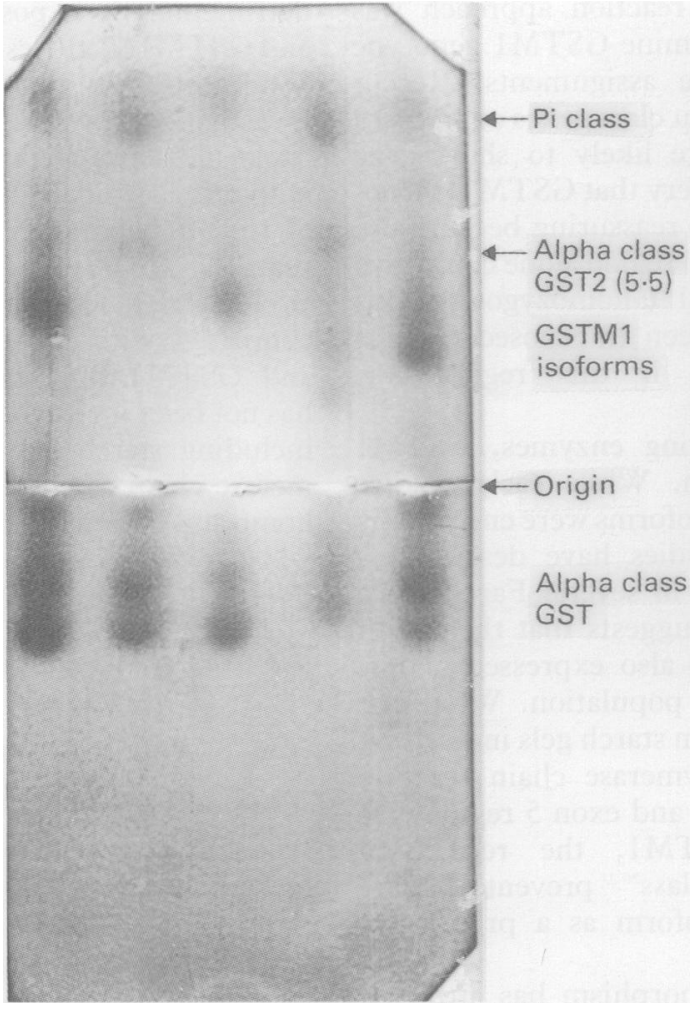

In all primary biliary cirrhosis cases and 68 of 69 controls, GSTM1 isoforms showed one of four distinct electrophoretic patterns; a fast anodal band, a slow anodal band, a three banded pattern, and a complete absence of detectable activity. These correspond with the GSTM1 B (GST1 1), GSTM1 A (GST1 2), GSTM1 A,B (GST1 2-1), and GSTM1 0 phenotypes respectively. In one control sample a band with an anodal mobility intermediate between GSTM1 B and GSTM1 A was seen (Fig 1). This may correspond with the previously reported GST1 3 phenotype. ${ }^{12}$ There was no evidence of heterodimers with GSTM1*A or GSTM1*B and because it is unlikely that the individual is a GSTM $1 \star 3$ homozygote, we propose a GSTM $1 \star 3 / G S T M 1 \star 0$ genotype.

The distribution of GSTM1 phenotypes in 44 patients with primary biliary cirrhosis and 69 control subjects is shown in the Table. The frequency of the various phenotypes, including GSTM1 0 and GSTM1 $\mathrm{A}$, in the two groups was similar $\left(\chi_{1}^{2}=0.2139\right.$ and $\chi_{1}^{2}=1 \cdot 22$ respectively, NS) and not different from that in published series. ${ }^{91012}$

GSTMI GENOTYPES IN CONTROLS AND PATIENTS WITH PRIMARY BILIARY CIRRHOSIS

The GSTM1 genotype in samples from five patients with primary biliary cirrhosis and five controls with the GSTM1 0 phenotype, and six patients with primary biliary cirrhosis and eight controls with GSTM1 expressing phenotypes was determined using the polymerase chain reaction. Figure 2 shows the presence of a $273 \mathrm{bp}$ fragment in individuals classified as possessing the GSTM1 gene and its absence in GSTM1 0 subjects. In all samples studied phenotype reflected genotype. The sample from the subject

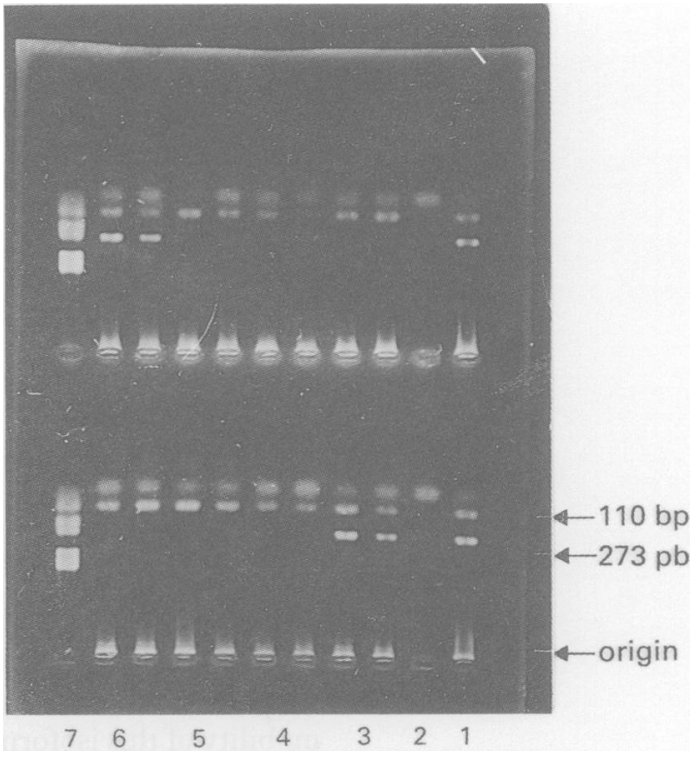

Figure 2: Determination of GSTM1 genotype using polymerase chain reaction. The agarose gel shows the $273 b p$ fragment amplified from the GSTM1 gene and the $110 \mathrm{bp}$ fragment from the $\beta$ globin gene. Samples were classified as GSTMI 0 genotype by the absence of the 273 bp fragment. Samples (bottom right to left) show; positive control target DNA for GSTMI and $\beta$ globin (1), negative control (no target DNA) (2), duplicate samples GSTM1 positive (3), GSTM1 negative genotypes $(4,5,6)$, and molecular weight markers ( $p$ BR322 Hae digest, Sigma) (7). Samples (top) show; 1, 2, 7 as below, 3, 4, 5, 6 patient samples.

who expressed the putative GSTM $1{ }^{\star} 3$ allele showed a positive GSTM1 genotype. The $110 \mathrm{bp}$ fragment amplified from the $\beta$ globin gene was identified in all subjects.

\section{Discussion}

We have described expression of GST in livers from controls and patients with aggressive primary biliary cirrhosis. Only patients with end stage disease were studied because the putatively protective effect of GSTM1 isoforms in mediating the damaging effects of inflammation should be most evident in this group. Inspection of isoenzyme patterns on starch gels, however, showed no obvious differences between controls and patients in the levels of expression of $\alpha, \mu$, and $\pi$ class GST. Furthermore, the incidence of GSTM1 0 was similar in the two groups. Apart from one individual with an apparently different phenotype, all subjects showed the expected GSTM1 0, GSTM1 A, GSTM1 B or GSTM1 A,B phenotypes.

Different groups have used various methods to determine GSTM1 phenotype. . $^{-11} 1+151819$ The starch gel electrophoresis approach used in this study has several advantages; for example, unlike most other methods, isoenzymes comprising the products of GSTM1*A, GSTM $1{ }^{\star} B$, and other genes such as GSTM $1{ }^{\star} 3$ can be identified and allele incidence determined. Furthermore, as separation is based on protein charge, phenotypes are not affected by changes in enzyme activity that result from circulating inhibitors or drug/diet effects on the level of gene expression. The technique, however, is not sufficiently sensitive to allow determination of GSTM1 phenotype in blood cells and depends on obtaining tissue samples in which phenotype could be influenced by disease. 
A polymerase chain reaction approach was therefore, used to determine GSTM1 genotype and confirm phenotype assignments. Recent studies have shown that $\mu$ class genes comprise a complex family that are likely to show gene conversion. ${ }^{26}$ Our discovery that GSTM1 phenotype reflects genotype is reassuring because use of primers to the exon $4 / 5$ region alone could give false positives in GSTM1*0 homozygotes if this part of the gene had been transposed or (less likely) false negatives if this region was polymorphic.

Like many detoxicating enzymes, GSTM1 shows multiple allelism. While early studies indicated that GSTM1 isoforms were encoded by three genes, recent studies have described a further allele, GST $1{ }^{\star} 3$, in several Far Eastern groups. ${ }^{12} 13$ This study suggests that this, or at least a similar allele, is also expressed at low frequency, in a British population. While the mobility of this isoform in starch gels indicates it is $\mu$ class and the polymerase chain reaction studies show the exon 4 and exon 5 regions are homologous with GSTM1, the recognised complexity of this GST class ${ }^{2627}$ prevents definite classification of this isoform as a product of GSTM1.

The GSTM1 0 polymorphism has attracted much recent interest because it seems to be associated with increased susceptibility to certain malignancies including lung, astrocytoma, stomach, and large bowel. ${ }^{11+16}$ The basis for this apparently increased susceptibility is unclear although it is believed that subjects with GSTM1 0 will show less effective detoxication of compounds with carcinogenic potential, such as epoxides and the products of reactive oxygen species attack on DNA, or both. In the context of liver disease, GSTM1 0 might be expected to be important as the gene is strongly expressed in the liver, contributing up to $50 \%$ of total GST activity in GSTM1 expressors. ${ }^{16}$ The finding of an increased occurrence of GSTM1 0 in patients with chronic hepatitis and alcoholic hepatitis supports this view although immunohistochemical studies in a small series of patients suffering hepatocellular carcinoma or cholangiocarcinoma do not indicate that the locus is protective in these malignancies. ${ }^{18}$

The suggested clinical usefulness of the GSTM1 0 polymorphism as a marker of susceptibility to disease depends on a greater knowledge of the function of these isoforms. Their putative role in the repair of oxidatively damaged DNA prompts the suggestion that expression of GSTM1 is important in the protection of cells from inflammation-derived reactive oxygen species. Subjects with GSTM1 0 may therefore, be more susceptible to inflammation induced damage which in some cases may progress to malignancy particularly following phagocyte mediated activation of potential carcinogens such as polycyclic aromatic hydrocarbons and aromatic amines. ${ }^{28}$ This study shows that even in patients with the most aggressive form of primary biliary cirrhosis, expression of GST isoforms is apparently unaltered and the failure to express GSTM1 does not seem to be disadvantageous.

While most interest has centred on the possibility that failure to express GSTM1 isoforms is detrimental, it is possible that the GSTM1*A and GSTM $1{ }^{\star} \mathrm{B}$ alleles are not equally protective. While this possibility has not been thoroughly investigated, we did not detect differences in allele incidence between controls and patients with primary biliary cirrhosis or adenocarcinoma of the stomach and colon. ${ }^{15}$ It is also possible that $\mathrm{GSTM} 1{ }^{\star} 0 / \mathrm{GSTM}{ }^{\star}{ }^{\star} \mathrm{A}$ and $\mathrm{GSTM}{ }^{\star}{ }^{\star} 0 /$ GSTM $1{ }^{\star} B$ heterozygotes are at increased risk compared with the corresponding GSTM1*A and GSTM1^B homozygotes. The possibility has not been investigated since most techniques, including starch gel electrophoresis and polymerase chain reaction, cannot distinguish these homo and heterozygotes.

We thank Dr John Taylor for much helpful advice regarding the $\mu$ family and the possibility that alleles are not equally protective, $\mathrm{Dr}$ Peter Jones and Dr Mary Jones for statistical analysis, and Mrs Julie Alldersea and Mrs Lei Zhao for the polymerase chain reaction analyses.

1 Watkins PB. Role of cytochromes $\mathrm{P}_{450}$ in drug metabolism and hepatotoxicity. Semin Liver Dis 1990; 10: 235-50.

2 Gonzalez FJ, Skoda RC, Kimura S, et al. Characterisation of the common genetic defect in humans deficient in debrisoquine. Nature 1988; 331: 442.

3 Mitchell SC, Waring RH, Haley CS, et al. Genetic aspects of the polymodally distributed sulphoxidation of S-carboxymethyl-L-cysteine in man. Br f Clin Pharmacol 1984; 18: 507-21.

4 Bousquet $\mathrm{O}$, Saigot T, Bernard F, et al. Hepatitis during treatment with tienilic acid. Therapie 1980; 35: 205-8.

5 Zimmerman HJ, Lewis HL. Ishak KG, Maddrey WC. Ticrynafen-associated hepatic injury: Analysis of 340 cases. Hepatology 1984; 4: 315-23.

6 Olomu AB, Vickers CR, Waring RH, et al. High incidence of poor sulphoxidation in patients with primary biliary cirrhosis. N Engl F Med 1988; 318: 1089-92.

7 Mannervik B, Alin P, Guthenberg C, et al. Identification of three classes of cytosolic glutathione S-transferase common to several mammalian species: Correlation between structural data and enzymatic properties. Proc Natl Acad Sci USA 1985; 82: 7202-6.

8 Hayes JD, Pickett CB, Mantle TJ, eds. Glutathione $S$ transferases and drug resistance. London: Taylor and Francis, 1990.

9 Board PG, Coggan M, Johnston P, Ross V, Suzuki T, Webb $G$. Genetic heterogeneity of the human glutathione transferases: a complex of gene families. Pharmacol Ther 1990; 48: 357-69.

10 Strange RC, Faulder GC, Davis B, et al. The human glutathione S-transferases: studies on the tissue distribution and genetic variation of the GST1, GST2 and GST3 isoenzymes. Ann Hum Genet 1984; 48: 11-20.

11 Seidegard J, Vorachek WR, Pero RW, Pearson WR. Heriditary differences in the expression of the human glutathione $\mathrm{S}$ transferase activity on trans-stilbene oxide are due to a gene transferase activity on trans-stilbene oxide are due to
deletion. Proc Natl Acad Sci USA 1988; 85: 7293-7.

12 Harada S, Abei M, Tanaka N, Agarwal DP, Goedde HW. Liver glutathione S-transferase polymorphism in Japanese and its $322-5$.

13 Bhattacharyya SP, Saha N, Wee KP. Glutathione S-transferase (GST) polymorphism among ethnic groups in Singapore with report of additional alleles at loci 1 and 2 . Gene Geography 1989; 3: 21-6.

14 Zhong S, Howie AF, Ketterer B, et al. Glutathione Stransferase mu locus: use of genotyping and phenotyping assays to assess association with lung cancer susceptibility. Carcinogenesis 1991; 12: 1533-7.

15 Strange RC, Matharoo B, Faulder GC, et al. The human glutathione S-transferases: a case-control study of the incidence of the GST1 0 phenotype in patients with adenocarcinoma. Carcinogenesis 1991; 12: 25-8.

16 Strange RC, Fryer AA, Matharoo B, et al. The human glutathione S-transferases: Comparison of isoenzyme expression in normal and astrocytoma brain. Biochim Biophys Acta 1992; 1139: 222-8.

17 Weincke JK, Kelsey KT, Lamela RA, Toscano WA. Human glutathione S-transferase deficiency as a marker of susceptibility to epoxide induced cytogenetic damage. Cancer Res 1990; 50: 1585-90.

18 Hayes PC, May L, Hayes JD, Harrison DJ. Glutathione Stransferases in human liver cancer. Gut 1991; 32: 1546-9.

19 Shea TC, Clafflin G, Comstock KE, et al. Glutathione transferase activity and isoenzyme composition in primary breast cancers. Cancer Res 1991; 50: 6848-53.

20 Ketterer B, Meyer DJ, Taylor JB, Pemble S, Coles B, Fraser G. GSTs and protection against oxidative stress. In: Hayes JD, Pickett CB, Mantle TJ, eds. Glutathione S-transferases and drug resistance. London: Taylor and Francis, 1990: 97109.

21 Strange RC, Fryer AA, Hiley C, Bell J, Cossar D, Hume R. Developmental expression of GST in human tissues. In:
Hayes JD, Pickett CB, Mantle TJ, eds. Glutathione $S$ - 
transferases and drug resistance. London: Taylor and Francis, $1990 ; 262-71$.

22 Afanas'eva IS, Spitsyn VA. Heriditary polymorphisms of glutathione S-transferase in the human liver in norma conditions and in alcoholic hepatitis. Genetika 1990; 26 1309-15.

23 Groppi A, Coutelle C, Fleury B, Iron A, Begueret J, Couzigou $P$. Glutathione S-transferase class mu in French alcoholic cirrhotic patients. Hum Genet 1991; 87: 628-30.

24 Maniatis T, Fritsch EF, Sambrook J, eds. Molecular cloning: a laboratory manual. New York: Cold Spring Harbour Laboratory, 1982: E.3-4.

25 Saiki RK, Gelfand DH, Stoffel S, Scharf SJ, Higuchi R, Horn
GT, et al. Primer-directed enzymatic amplification of DNA with a thermostable DNA polymerase. Science 1988; 239: with a thermostable DNA polymerase. Science 1988, 239 :

26 Taylor JB, Oliver J, Sherrington R, Pemble SE. Structure of human glutathione $\mathrm{S}$-transferase class Mu genes. Biochem $\mathcal{F}$ 1991; 274: 587-93.

27 DeJong JL, Mohandas T, Tu C-PD. The human Hb (MU class glutathione $\mathrm{S}$-transferases are encoded by a dispersed gene family. Biochem Biophys Res Commun 1991; 180: 1522 .

28 Weitzman SA, Gordon LI. Inflammation and cancer: role of phagocyte-generated oxidants in carcinogenesis. Blood 1990; 76: 655-63. 